\title{
Preparing for the unexpected; A reflection on the Hospital Preparedness for COVID-19
}

\author{
Jayasekera $P^{1}$, Navaratne $V^{2}$, Govindapala $D^{1}$, Nakkawita $D^{2}$, Gamage $A^{3}$ \\ Journal of the Ceylon College of Physicians, 2020, 51, 68-70
}

\section{Introduction}

Pandemics and outbreaks have swept across the globe claiming lives of many and often causing significant social and economic impact. Whether mild, moderate or severe, pandemics affect a large proportion of the global population and require a multisectoral response to curtail it. Planning for and responding to a pandemic is complex as public health officials, healthcare professionals, researchers, and scientists across the world need to work together for several months or years to prevent and control a pandemic ${ }^{1}$.

In early December 2019, an outbreak of a novel corona virus (SARS-CoV-2) was reported in Wuhan, China and a Public Health Emergency Concern was declared by the Emergency Committee of the World Health Organisation (WHO) on the $30^{\text {th }}$ January $2020^{2}$. The first case of COVID-19 was reported from Sri Lanka on the $27^{\text {th }}$ of January 2020, a tourist from Hubei Province, China ${ }^{3}$. After six weeks, the first Sri Lankan was diagnosed with COVID-19 on the $10^{\text {th }}$ of March 2020 and the number has continued to rise since then ${ }^{4}$. There were more than 3.7 million confirmed cases worldwide with over 259,474 deaths reported as of 9th May, and 835 laboratory confirmed cases with nine deaths have occurred in Sri Lanka by this date ${ }^{5}$. We present an unexpected case finding among our own staff while preparing for the pandemic.

\section{Strategic plan to mitigate the impact}

Since the first reported case of COVID-19 in Sri Lanka, the University Hospital, Kotelawala Defence
University (UHKDU) started preparing for the possible influx of infected patients. The main focus of the preparedness has been on clinical detection, management of patients, prevention of further transmission of the disease, and minimising risk of infection to the health care workers.

A triage system was established at the entrance to the hospital and specific areas were designated to manage COVID-19 patients. A separate respiratory unit was established in an isolated area within the hospital premises to evaluate suspected COVID-19 patients and all required measures were taken to mitigate the nosocomial transmission of the disease. Regular training programmes were conducted for the health care workers on proper donning and doffing of personal protective equipment (PPE), infection prevention tenets, sample collection, and patient management. The rational use of PPE was emphasized to all health care workers, and the rate of PPE use was actively monitored. The hospital staff was divided into three teams, and extended working hours were introduced to limit the total number of health care personnel involved in patient care at any given time. Non-urgent and elective surgical procedures were cancelled to reduce the hospital admissions, and visitors were restricted to the hospital. A dedicated multidisciplinary team was established to ensure the smooth running of this preparedness program and to provide a sufficient supply of PPE and medicines.

However, at the outset, our preparedness program did not include clear plans outlining the management

\footnotetext{
${ }^{1}$ Department of Medicine, ${ }^{2}$ Department of Microbiology, ${ }^{3}$ Department of Public Health, General Sir John Kotelawala Defence University, Sri Lanka.
}

*Correspondence: PJ e-mail: priyamja@yahoo.com

http://orcid.org/0000-0002-6699-7937

Received 28 April 2020, accepted 15 May 2020.

This is an open-access article distributed under the terms of the Creative Commons Attribution License, which permits unrestricted use, distribution, and reproduction in any medium, provided the original author and source are credited. 
of health care workers (HCWs) regarding work restrictions or quarantine requirements following an exposure to COVID-19 patients. At this stage, the possibility of a staff member contracting the disease was considered to be remote as there were no confirmed cases in our health care facility as well as due to the absence of community transmission of COVID-19 in Sri Lanka. Hence, the HCWs with mild respiratory symptoms without any contact or exposure history were not excluded from work. Further, social distancing and other standard transmission-based precautions were not fully adhered to, by the HCWs during their interactions with one another.

\section{Unexpected COVID-19 positivity among hospital staff}

On the $23^{\text {rd }}$ of March, a nursing officer and a senior administrative officer of the hospital were evaluated for possible COVID-19. At the time of presentation, apart from suggestive respiratory symptoms, they both were not fulfilling the criteria for PCR testing according to the guidelines issued by the Ministry of Health. However, the test was performed due to two main reasons. Firstly, because there had been another staff member in the same unit with suggestive symptoms and a significant exposure history, a few days prior to their presentation. Secondly, during this period. there were reported cases of COVID-19 infection in other hospitals in the Colombo district.

Both of the staff members were tested positive for COVID-19 on the same day, resulting an unprecedented crisis in the hospital.

The main challenges we faced were:

(1) Detection of the original source of infection and the epidemiological links

(2) Making quarantine decisions on the exposed HCWs without national or institutional guidelines being available

(3) Functioning of the hospital with limited human and other resources

(4) Mitigation of further nosocomial transmission of the disease

The imposition of curfew on the same week had a massive impact on return-to-work of nonquarantined staff, making the existing situation worse.

\section{Outbreak response at the hospital}

Many staff members did not report to work on the following morning after the case detection, claiming that they had contacts with the two positive cases. Some local public health authorities started to place the HCWs on quarantine in their respective areas. The need to identify the staff who were exposed and required quarantine was deemed essential in order to ensure smooth running of the hospital. A team consisting of physicians, microbiologists, community physicians, psychologists and hospital administrators were appointed to define the exposure risks and make quarantine decisions. Those exposure risk reviews were time intensive and the decisions taken by the hospital appointed committee were not accepted by some of the local public health authorities.

Adding to the burden further, there were no local guidelines on exposure risk categorization at this point of time. Owing to lack of a clear definition of an "exposed case" 6 the team had to gather a lot of information from the two employees who were tested positive for COVID-19 and from all the staff members who had contact with them. The Epidemiology Unit was consulted for case definitions and further guidance. The Ministry of Health has issued a preliminary guideline on post exposure risk assessment of the HCWs on the third day of the incident ${ }^{7}$. The collaborative efforts of the hospital team and the Ministry of Health were crucial in averting the calamity.

The overestimation of the risk by the society and its panic response had resulted in denial of housing for both exposed and non-exposed healthcare workers. Hence hospital authorities had to sort out their accommodation and other needs. A separate unit was established for the HCWs who were placed on quarantine and they were closely observed for the development of symptoms. Some employees were quarantined at their homes and the details were shared with the respective local health authorities. Provisions were made to monitor them closely.

\section{Mitigating spread within the hospital}

The existing infection prevention measures were further strengthened after detection of two staff members with COVID-19. Repeated awareness programs on standard precautions, contact and droplet precautions and rational use of PPE were conducted by the microbiologists and infection prevention and control (IPC) team for the staff. Simultaneously, an 
institutional guideline was prepared on the use of PPE and IPC measures. Proper distribution of PPE in the context of shortages and with increased demands from the staff was a major challenge faced by the infection control unit of the hospital. In-house production of some of the PPE was initiated in order to circumvent the situation.

\section{Psychological and other support extended to the staff}

The hospital staff was continuously working long and irregular hours since the onset of the pandemic, contributing to an increased level of stress and burnout. The major issues contributed to development of stress included (1) fear of contracting the disease due to initial exposure (2) fear of subsequent exposures to positive patients (3) stigma and social withdrawal related to disease positivity and (4) fear of being isolated during quarantine. The daunting task at hand was to relieve their stress and get them back to normal work routine.

The following actions were taken to address these issues; (1) Testing staff with mild respiratory symptoms for SARS CoV-2. The fact that none of them became positive for COVID-19, alleviated the fear of contracting the disease to a certain extent. (2) Repeated teaching, advice, guidelines on rational use of PPE, and 24-hour helpline. (3) Quarantine in the newly arranged in-house observation wards which consisted of an in-house quarantine unit. Further, the daily visits to the quarantined staff by medical staff helped them to relieve their anxiety and stress.

This situation created an acute and significant shortage in the workforce. Shortage in PPE made a significant psychological impact on clinicians and other staff. A team of doctors from armed forces and an enthusiastic, energetic in-house team of nurses and health care assistants, restored the functionality of the hospital. Supplementation of PPE from the Ministry of Health, donations from well-wishers, and in-house preparing of items relieved the shortage and the level of stress.

\section{Conclusions}

The threat of this pandemic will linger until it is eliminated globally. Until such time, all activities related to outbreak response should continue with regular review of the process and making of necessary adjustments when needed. Prompt implementation of infection-control policies in hospitals when cases are identified in the community could potentially prevent hospital outbreaks. The need for rapid response and outbreak control at a health care centre is evident from the large numbers of HCWs affected by this outbreak, the impact on patients, and the disruption of patient care. It is therefore mandatory to have a rapid response plan in which financial costs too have to be considered.

\section{References}

1. Planning and Preparedness Resources, Pandemic Influenza (Flu), Centres for Disease control and Prevention, USA. https://www.cdc.gov/flu/pandemic-resources/planningpreparedness/index.html. Accessed May 13, 2020.

2. Statement on the second meeting of the International Health Regulations (2005) Emergency Committee regarding the outbreak of novel coronavirus (2019-nCoV). https:// www.who.int/news-room/detail/30-01-2020-statement-onthe-second-meeting-of-the-international-health-regulations(2005)-emergency-committee-regarding-the-outbreak-ofnovel-coronavirus-(2019-ncov). Accessed May 13, 2020.

3. Epidemiology Unit Sri Lanka. (2020). COVID-19 situation report 28/01/2020. [Online] Available at: http:// www.epid.gov.lk/web/index.php?option=com_content \&view=article\&id=225\&lang=en Accessed 22 April 2020

4. Epidemiology Unit Sri Lanka. (2020). COVID-19 situation report 11/03/2020. [Online] Available at: http:// www.epid.gov.lk/web/index.php?option=com_content \&view=article\&id=225\&lang=en [Accessed 22 April 2020]

5. Epidemiology Unit Sri Lanka. (2020). COVID-19 situation report 9/05/2020. [Online] Available at: http:// www.epid.gov.lk/web/index.php?option=com_content \&view=article\&id=225\&lang=en [Accessed 22 April 2020]

6. World Health Organization. (2020). Health workers exposure risk assessment and management in the context of COVID-19 virus: interim guidance, 4 March 2020. World Health Organization. https://apps.who.int/iris/handle/10665/ 331340 